\title{
Spin-phonon coupling in highly correlated transition-metal monoxides
}

\author{
Ch. Kant ${ }^{\mathrm{a}}$, F. Mayr, T. Rudolf, M. Schmidt, F. Schrettle, J. Deisenhofer, and A. Loidl \\ Experimental Physics V, Center for Electronic Correlations and Magnetism, University of Augsburg, \\ 86135 Augsburg, Germany
}

\begin{abstract}
We report on the optical response of the highly correlated transitionmetal monoxides $\mathrm{MnO}, \mathrm{FeO}, \mathrm{CoO}$ and $\mathrm{NiO}$ in the far-infrared regime. The main focus is put on spin-phonon coupling effects, which are found to significantly influence the lattice dynamics in the magnetically ordered phase. Measurements have been performed in the ordered and paramagnetic state for temperatures up to $550 \mathrm{~K}$. A clear splitting of the cubic mode accompanying the transition into long range magnetic order can be identified in $\mathrm{MnO}, \mathrm{CoO}$ and $\mathrm{NiO}$. In the case of $\mathrm{FeO}$ it is argued that an anisotropic phonon response seems to be very likely, though it could not be observed directly. The results are compared to recent experimental and theoretical studies in frustrated magnets, which predict the splitting of zone center phonon modes induced by a non-cubic spin-density distribution.
\end{abstract}

\section{Introduction}

For decades transition-metal monoxides (TMO) have been attracting considerable attention in condensed matter science. Starting with the first direct observation of antiferromagnetism in $\mathrm{MnO}$ by Shull and Smart [1], a state which was previously predicted by Néel [2], and a controversial debate on the origin of insulating ground states triggered by de Boer and Verwey [3] and Mott [4], this class of oxides has stimulated the development of modern solid state physics substantially. Basic electronic and magnetic properties can still not be described satisfactorily from the theoretical point of view $[5,6]$. Besides that, TMOs are regarded as model systems for spin-phonon interaction due to their conceptual simple crystal structure. The idea of a purely magnetic-order induced anisotropy in the phonon properties has been put forth by Massidda et al. [7] and was recently reinvestigated by Luo et al. [8]. This interest was further stimulated by the observation of transverse optical (TO) phonon modes splitting right at the onset of long range magnetic ordering in $\mathrm{MnO}$ and $\mathrm{NiO}$ by neutron diffraction [9] and in several cubic spinel compounds [10-14] probed by means of optical spectroscopy. In [15] it could be impressively demonstrated that this magnetic order induced phonon splitting can be shifted towards $0 \mathrm{~K}$ in $\mathrm{ZnCr}_{2} \mathrm{Se}_{4}$ by applying moderate external magnetic fields. Hence, this effect was interpreted in terms of a spin-driven Jahn-Teller effect [16] undergoing a spin-Peierls like transition at the magnetic ordering $[17,18]$.

In principal, lattice distortions accompanying the phase transitions can explain the splitting of the phonon modes due to the reduced lattice symmetry. However, on the basis of local spindensity approximations (LSDA) it was clearly stated in [7] and [8] that the phonon anisotropy can be explained solely by the non-cubic spatial arrangement of the magnetic moments in the late TMOs, neglecting any structural distortions. Furthermore, it was pointed out there in

\footnotetext{
a e-mail: christian.kant@physik.uni-augsburg.de
} 
Table 1. Structural and magnetic properties of the TMOs. $a$ is the lattice constant at room temperature, $T_{\mathrm{N}}$ the Néel temperature and $\Theta_{\mathrm{CW}}$ the Curie-Weiss temperature. Also provided are the lattice symmetry below $T_{\mathrm{N}}$ together with the observed spin directions. The properties of FeO depend on the exact stoichiometry.

\begin{tabular}{|c|c|c|c|c|}
\hline & $\mathrm{MnO}$ & $\mathrm{FeO}$ & $\mathrm{CoO}$ & $\mathrm{NiO}$ \\
\hline$a(\AA)$ & $4.445^{a}$ & $\approx 4.3^{d}$ & $4.255^{g}$ & $4.1899^{m}$ \\
\hline$T_{\mathrm{N}}(\mathrm{K})$ & $118^{a}$ & $\approx 200^{e}$ & $290^{i}$ & $523^{n}$ \\
\hline$\Theta_{\mathrm{CW}}(\mathrm{K})$ & $-548^{b}$ & $\approx-136^{f}$ & $-330^{j}$ & $-2000^{j}$ \\
\hline symmetry $T<T_{\mathrm{N}}$ & rhomb. $^{a}$ & rhomb. ${ }^{g}$ & monocl. ${ }^{k}$ & rhomb. ${ }^{m}$ \\
\hline spin direction & {$\left[\begin{array}{lll}1 & 1 & \overline{2}\end{array}\right]^{c}$} & {$\left[\begin{array}{lll}1 & 1 & 1\end{array}\right]^{h}$} & {$\left[\begin{array}{lll}\overline{1} & \overline{1} & 7\end{array}\right]^{l}$} & {$\left[\begin{array}{lll}1 & 1 & \overline{2}\end{array}\right]^{o}$} \\
\hline${ }^{a}$ Morosin $[23]$ & \multicolumn{2}{|c|}{${ }^{b}$ Tyler $[24]$} & \multicolumn{2}{|c|}{${ }^{c}$ Goodwin $[25]$} \\
\hline${ }^{d}$ McCammon and Liu [26] & \multicolumn{2}{|c|}{${ }^{e}$ McCammon $[21]$} & \multicolumn{2}{|c|}{${ }^{f}$ Koch and Fine $[27]$} \\
\hline$g$ Tombs and Rooksby [28] & \multicolumn{2}{|c|}{${ }^{h}$ Shull et al. $[29]$} & \multicolumn{2}{|c|}{${ }^{i}$ Bizette $[30]$} \\
\hline${ }^{j}$ Singer $[31]$ & \multicolumn{2}{|c|}{${ }^{k}$ Saito et al. $[32]$} & \multicolumn{2}{|c|}{${ }^{l}$ Roth $[19]$} \\
\hline${ }^{m}$ Bartel and Morosin [33] & \multicolumn{2}{|c|}{${ }^{n}$ Rooksby [34] } & \multicolumn{2}{|c|}{${ }^{\circ}$ Kondoh and Takeds } \\
\hline
\end{tabular}

particular that the distortions in $\mathrm{MnO}$ can account for a splitting of the order of $1 \%$ only, being significantly lower than the influence of the former effect.

All compounds subject of the present study share common structural and magnetic properties. $\mathrm{MnO}, \mathrm{FeO}, \mathrm{CoO}$ and $\mathrm{NiO}$ crystallize in the cubic rock-salt structure (space group $F m \overline{3} m$ (\#225) with occupied Wyckoff positions $4 a$ and $4 b$ ) in the paramagnetic state. In this structure two triply degenerate $T_{1 u}$ phonon modes are expected from group symmetry arguments. While one accounts for the acoustical branch and consequently is not detectable in the optical experiments, the other is IR active and represents the vibration where both sublattices, formed by the two different types of ions, oscillate against each other [8]. The magnetically ordered phase can be characterized by a type-II antiferromagnet. Thus the ordered spin pattern can be described by ferromagnetic (111) sheets stacking antiferromagnetically along [111] [19]. The direction of the magnetic moments, however, is non-generic. Some structural and magnetic properties are compiled in Table 1 together with the corresponding references. The stoichiometric composition of $\mathrm{FeO}$ does not establish a homogenous and stable phase [20]. Wüstite exists in an iron deficient crystalline structure $\mathrm{Fe}_{1-\delta} \mathrm{O}$ in the range $0.02 \leq \delta \leq 0.12$ [21]. Its physical properties depend on the exact stoichiometry. Going from $\mathrm{MnO}$ to $\mathrm{NiO}$ the lattice constant decreases owing to the shrinking ionic radii of the metal ions. Most remarkable is the continuous increase of the ordering temperatures, while $\Theta_{\mathrm{CW}}$ decreases rapidly with the exception of the iron compound. In the ordered phase the lattice symmetry reduces to rhombohedral for $\mathrm{MnO}, \mathrm{FeO}$ and $\mathrm{NiO}$, only in $\mathrm{CoO}$ a monoclinic structure has been identified. However, it has been pointed out, that the spin structure of $\mathrm{MnO}$ is not compatible with this assignment and should be of lower symmetry [22].

Concerning the electronic structure, in the TMOs the $3 d$-ion is located in an octahedral environment of oxygen ions. Hence, the fivefold degenerate $d$-levels are split into a lower $t_{2 g}$ triplet and an upper $e_{g}$ doublet. As all $3 d$ ions under consideration exhibit high-spin configurations and strong Hund's-rule coupling, the resulting spin states are $S=5 / 2$ for $\mathrm{MnO}, S=2$ for $\mathrm{FeO}, S=3 / 2$ for $\mathrm{CoO}$ and $S=1$ for NiO. Significant spin-orbit coupling is expected for the iron and cobalt compound, whereas it should be negligible for $\mathrm{MnO}$ revealing a half-filled $d$-shell and also for $\mathrm{NiO}$ with a filled $t_{2 g}$ triplet and a half-filled $e_{g}$ doublet.

Several studies probing the lattice dynamics in the TMOs are available in literature. For $\mathrm{MnO}$ inelastic neutron scattering experiments have been reported in [9] giving first evidence for a spitting of the zone center TO mode. Optical reflectivity spectra of the far infrared (FIR) response in $\mathrm{MnO}$ at room temperature have been published in [36] and [37]. Recently, some of the authors have reported on the temperature dependence, having identified three distinct oscillators at $5 \mathrm{~K}[38]$. FeO was investigated by means of optical spectroscopy in the FIR domain first by Bowen et al. [39] and Prévot et al. [40]. Spectra recorded at various temperatures 
are also provided in [41] and recently a pressure dependent study up to 33 GPa has been performed [42]. However, no systematic analysis of the temperature dependent evolution of the phonon modes has been reported yet. The phonon dispersion in the whole Brillouin-zone has been studied in [43] by inelastic neutron scattering. Several optical studies were performed on CoO. First reflectivity spectra at ambient conditions were recorded by Gielisse et al. [44]. Absorption measurements revealed additional weak excitations in the FIR domain [45] at low temperatures. Recently some of us have published a comprehensive temperature dependent study of the phonon and low lying electronic excitations, which could be interpreted as transitions between the spin-orbit and molecular field split ground state [46]. Furthermore, a splitting of the transverse optical (TO) mode has been identified below $T_{\mathrm{N}}$. Neutron scattering measurements focussing on the phonon and low lying magnetic excitations have been reported in [47] and recently in [48]. As to the optical properties of $\mathrm{NiO}$, they were first investigated in a broad frequency range by Newman and Chrenko [49]. First temperature dependent measurements from 300 to $1273 \mathrm{~K}$ have been performed by Mochizuki and Satoh in [50]. The neutron scattering data in [9] indicate a splitting of the TO phonon at low temperatures.

To summarize the experimental situation for $\mathrm{MnO}, \mathrm{FeO}, \mathrm{CoO}$ and $\mathrm{NiO}$ regarding the optical properties, only few systematic temperature dependent investigations are available and a direct comparison has not yet been drawn. The purpose of the present work is to continue our efforts in understanding the interplay of the spin degrees of freedom and the lattice dynamics for the late TMOs. Therefore, we performed reflectivity measurements from $T \approx 5 \mathrm{~K}$ up to the respective paramagnetic states for all compounds.

The outline of the present paper is as follows: After reporting the magnetic susceptibility and specific heat data obtained from the investigated samples in order to characterize the quality in Sec. 2, reflectivity spectra for all compounds are provided in Sec. 3, and subsequently the phonon properties are analyzed as a function of temperature. Finally, these results are compared and discussed in Sec. 4.

\section{Experimental details and sample characterization}

All investigated TMOs were purchased commercially. Plate-like single crystals with surfaces of approximately $5 \times 5 \mathrm{~mm}^{2}$ polished to optical quality and various thicknesses ranging from 1 to $5 \mathrm{~mm}$ were obtained from MaTeck $\mathrm{GmbH}(\mathrm{MnO}, \mathrm{FeO}$ and $\mathrm{NiO}$ ) and CrysTec $\mathrm{GmbH}$ $(\mathrm{CoO})$, respectively. The crystals were cut parallel to $\left(\begin{array}{lll}1 & 0 & 0\end{array}\right)$ planes except for CoO, which offers a (110) orientation. Characterization of the sample qualities were ensured by inspecting the magnetic and calorimetric properties in a wide temperature range. The magnetic susceptibilities $\chi(T)$ were obtained for $1.8 \leq T \leq 400 \mathrm{~K}$ utilizing a commercial superconducting quantum interference device magnetometer (Quantum Design MPMS-5) and the heat capacities were studied via a Quantum Design physical properties measurement system between 2 and $305 \mathrm{~K}$. Reflectivity spectra were recorded using the Bruker Fourier-Transform spectrometers IFS 113v and IFS 66v/S for frequencies ranging from 50 to at least $8000 \mathrm{~cm}^{-1}$. Both spectrometers are equipped with a He-flow (4-600 K) cryostat. The broad frequency range allows to determine $\varepsilon_{\infty}$ unambiguously and enables the application of the Kramers-Kronig relation. For this analysis a constant continuation of the reflectivity was applied towards low frequencies and a smooth power law behavior was assumed at the high-frequency limit. However, due to the finite conductivity in FeO, a Drude-like extrapolation had to be chosen at elevated temperatures for the lower frequency boundary.

The magnetic susceptibilities for $\mathrm{MnO}, \mathrm{FeO}$ and $\mathrm{CoO}$ obtained from the investigated samples are documented in Fig. 1. For the nickel compound these data are not plotted, since the ordering temperature amounts to $T_{\mathrm{N}}=523 \mathrm{~K}$. The results are in good agreement with previous studies $[27,51,52]$. Cooling the samples from elevated temperatures the susceptibilities increase monotonically in the paramagnetic state obeying Curie-Weiss behavior. Each compound develops a cusp at $T_{\mathrm{N}}$ indicating the onset of long range antiferromagnetic (AFM) ordering. The transition temperatures amount to $T_{\mathrm{N}}=118,198$ and $289 \mathrm{~K}$ for $\mathrm{MnO}, \mathrm{FeO}$ and $\mathrm{CoO}$, respectively, and correspond nicely to reported values $[21,23,30]$. However, a minor impurity phase can 


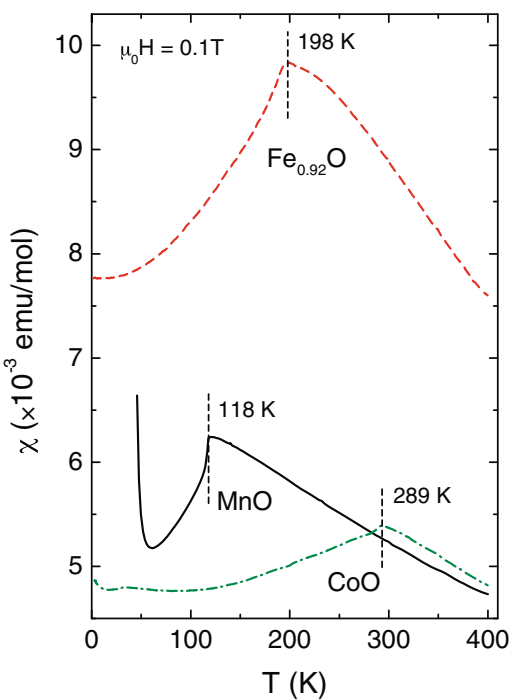

Fig. 1. Magnetic susceptibility between 1.8 and $400 \mathrm{~K}$ for the investigated samples $\mathrm{MnO}, \mathrm{FeO}$ and $\mathrm{CoO}$. The measurements were done in externally applied field of $\mu_{0} H=0.1 \mathrm{~T}$. At low temperatures $\mathrm{MnO}$ is dominated by ferrimagnetic $\mathrm{Mn}_{3} \mathrm{O}_{4}$ inclusions. The hump close to $30 \mathrm{~K}$ in the CoO data can be attributed to $\mathrm{Co}_{3} \mathrm{O}_{4}$.

be detected in $\mathrm{MnO}$ and $\mathrm{CoO}$, which are well known to occur during the single crystal growth and can hardly be avoided $[52,53]$. Inclusions of the ferrimagnetic oxide $\mathrm{Mn}_{3} \mathrm{O}_{4}$ dominate the behavior below $T=50 \mathrm{~K}$ in the $\mathrm{MnO}$ sample. From the magnetization at lowest temperatures (not shown in Fig. 1), its amount can be estimated to be about 3.5\%. A slight hump in the CoO data located at approximately $30 \mathrm{~K}$, can be attributed to the tetravalent cobalt oxide, which orders antiferromagnetically at $T_{\mathrm{N}}=29.5 \mathrm{~K}$ [54]. From fits of the paramagnetic susceptibility to a Curie-Weiss behavior

$$
\chi(T)=\frac{C}{T-\Theta_{\mathrm{CW}}},
$$

the Curie constant $C$ and the Curie-Weiss temperature $\Theta_{\mathrm{CW}}$ can be obtained. The results are compiled in Table 2 and agree fairly well with literature. We find good agreement in the magnetic ordering temperatures, while $\Theta_{\mathrm{CW}}$ seems to be systematically enhanced in our analysis. From the Curie constants we calculated effective paramagnetic moments $p_{\text {eff }}$ and in addition

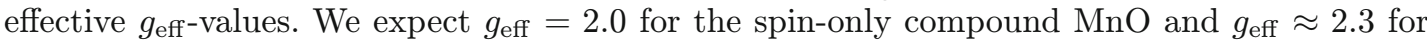
$\mathrm{FeO}$ and $\mathrm{CoO}$ with significant spin-orbit coupling. The experimental results as documented in Table 2 are significantly enhanced. But obviously, strictly reliable results of $\Theta_{\mathrm{CW}}$ and the effective paramagnetic moments can only be achieved by extending the magnetic susceptibility measurements to much higher temperatures $\left(T \gg T_{\mathrm{N}}\right)$.

Table 2. Magnetic properties of the investigated samples as derived from the susceptibility data. The spin-only effective moments $2 \sqrt{S(S+1)}$ and the effective $g_{\text {eff }}$ values are also given. In the analysis of $\mathrm{FeO}$ we took the non-stoichiometric with $\delta=0.08$ into account.

\begin{tabular}{cccc}
\hline & $\mathrm{MnO}$ & $\mathrm{FeO}$ & $\mathrm{CoO}$ \\
\hline$T_{\mathrm{N}}(\mathrm{K})$ & 118 & 198 & 289 \\
$\Theta_{\mathrm{CW}}(\mathrm{K})$ & -675 & -245 & -450 \\
$C\left(\frac{\mathrm{emu} \mathrm{K}}{\mathrm{mol}}\right)$ & 5.08 & 4.22 & 4.09 \\
$p_{\text {eff }}\left(\mu_{B}\right)$ & 6.36 & 6.30 & 5.70 \\
$2 \sqrt{S(S+1)}$ & 5.91 & 4.90 & 3.87 \\
$g_{\text {eff }}$ & 2.15 & 2.57 & 2.94 \\
\hline
\end{tabular}




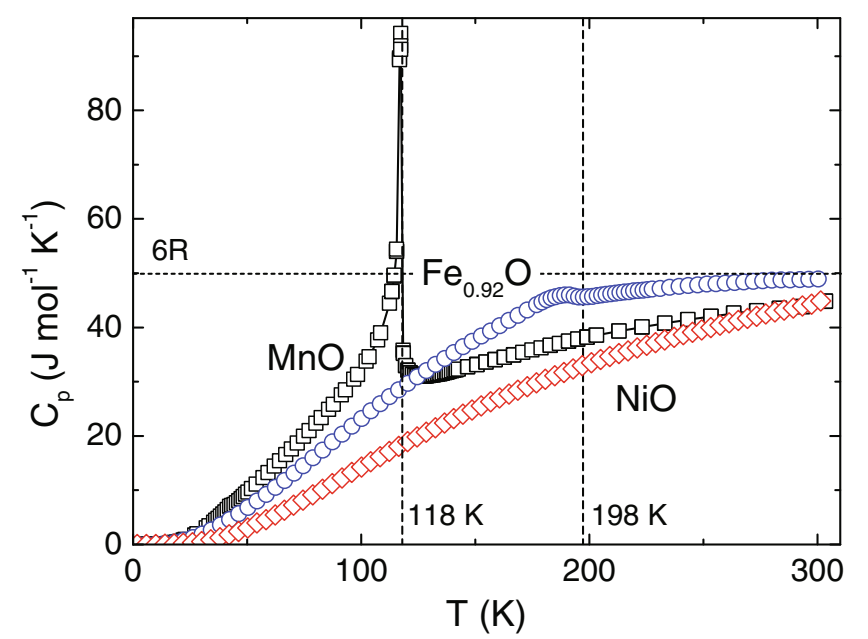

Fig. 2. Specific heat of $\mathrm{MnO}, \mathrm{FeO}$ and $\mathrm{NiO}$ as a function of temperature. Vertical dashed lines indicate magnetic ordering temperatures of $\mathrm{MnO}(118 \mathrm{~K})$ and $\mathrm{FeO}(198 \mathrm{~K})$.

The temperature dependence of the molar heat capacities $C_{p}$ for $\mathrm{MnO}, \mathrm{FeO}$ and $\mathrm{NiO}$ are displayed in Fig. 2. The data for $\mathrm{CoO}$ will be further analyzed later on and are presented in the corresponding section. The AFM transitions can clearly be identified as $\lambda$-like anomalies for $\mathrm{MnO}$ and $\mathrm{FeO}$ and agree well with the findings of the susceptibility measurements. For NiO the specific heat increases monotonically in a featureless manner and approaches $45 \mathrm{~J} / \mathrm{mol} \mathrm{K}$ at room temperature. Thus, it is slightly lower than the estimated value of $6 R$ expected for a lattice with six phonon branches from Dulong-Petit's law. Comparing these data to $C_{p}$ of the isostructural $\mathrm{MnO}$, it becomes apparent that the latter are clearly enhanced around the ordering temperature due to magnetic contributions, but almost coincide with the former at higher temperatures. In case of the iron monoxide the full expected heat capacity is nearly reached at the highest measured temperatures. However, since low lying electronic levels are present in $\mathrm{FeO}$, introduced by the splitting of the ground term due to spin-orbit interaction, some contributions to $C_{p}$ can be expected from a Schottky-like anomaly.

Further characterizing measurements were performed for $\mathrm{Fe}_{x} \mathrm{O}$, since it occurs in a broad variety of stoichiometries with $x$ ranging from 0.88 to 0.98 [21]. From x-ray diffraction experiments on the crushed single crystal a refined lattice constant of $a=4.298 \AA$ could be evaluated, which corresponds to an iron deficiency of $\delta=0.08$ per formula unit. Thus, the exact stoichiometry of the investigated sample is found to be $\mathrm{Fe}_{0.92} \mathrm{O}$; however, for shortness this quantitative labeling will be dropped in the following.

Though a minor impurity phase was identified in $\mathrm{MnO}$ and $\mathrm{NiO}$, bulk sensitive reflectivity measurements should mainly probe the optical properties of the dominant phase. No attempts were undertaken to improve the sample quality by means of annealing in the appropriate transition-metal vapors as proposed for polycrystalline samples or small single crystals $[52,53]$.

\section{Results and analysis}

Figure 3(a)-(d) compares the reflectivity spectra of $\mathrm{MnO}, \mathrm{FeO}, \mathrm{CoO}$ and $\mathrm{NiO}$ in the FIR regime for $100 \leq \omega \leq 800 \mathrm{~cm}^{-1}$. Displayed are the corresponding data obtained at low and room temperatures, respectively, which is above and below the Néel temperatures except for NiO. The optical response is dominated by a broad reststrahlen band in each case predicted by symmetry arguments for the cubic rock-salt structure. At the $\Gamma$ point the transverse optical phonons lead to a strong increase of the reflectivity, while the longitudinal optical (LO) modes are separated due to the non-vanishing polarization associated with the vibration. For an ideal ionic solid with negligible damping, these characteristic eigenfrequencies are determined by both edges of 


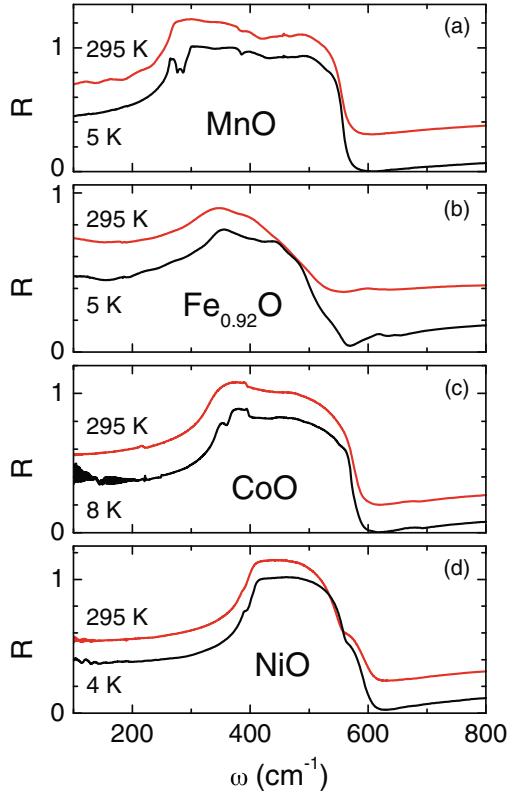

Fig. 3. Reflectivity spectra of (a) $\mathrm{MnO}$, (b) $\mathrm{FeO}$, (c) $\mathrm{CoO}$ and (d) $\mathrm{NiO}$ for frequencies between 100 and $800 \mathrm{~cm}^{-1}$. The upper curves in each frame are shifted by an amount of 0.2 for clarity and correspond to the results at ambient conditions, while the lower curves were obtained at low temperatures.

the reststrahlen band, where the reflectivity is close to unity. Deviations from this behavior can principally be attributed to the finite lifetime of the oscillations, leading to a broadening of the band, and to multiple phonon processes. The latter mechanism involves zone boundary optical and acoustical modes summing up to an IR-active zero wave-vector excitation [55]. They are usually weaker in intensity and exhibit a similar temperature shift as the main resonances. In this context the humps on top of the reflectivity plateau at about $500 \mathrm{~cm}^{-1}$ in $\mathrm{MnO}$ and close to $400 \mathrm{~cm}^{-1}$ in $\mathrm{CoO}$ have been mentioned $[38,46]$. Also the shoulder located at $\approx 560 \mathrm{~cm}^{-1}$ in the spectrum of $\mathrm{NiO}$ was interpreted in this manner [50]. Indeed, the two-phonon density of states calculated in [56] revealed weak maxima close to these frequencies. However, excitations, predicted to be much stronger, cannot be identified in the spectra unambiguously. Besides the structures superimposing the reststrahlen band, additional features can be identified from the raw data located in $\mathrm{FeO}$ at $600 \mathrm{~cm}^{-1}$, in $\mathrm{CoO}$ at $220 \mathrm{~cm}^{-1}$ and $680 \mathrm{~cm}^{-1}$ and in $\mathrm{NiO}$ a tiny anomaly is visible close to $390 \mathrm{~cm}^{-1}$. It should also be mentioned that for $\mathrm{FeO}$ the reflectivity increases significantly towards low frequencies contrarily to the observations in the other TMOs. This increase in reflectivity for $\omega \rightarrow 0$ indicates the existence of free charge carriers.

On cooling the reflectivity of all samples increases slightly. While all resonances tend to become more pronounced signaling an increasing lifetime, additional oscillations occur when passing the magnetic ordering transition and are clearly visible at the low frequency edge of the reststrahlen bands in Fig. 3 with the exception of FeO. For the manganese oxide at least three distinct oscillators develop from the main mode. In $\mathrm{FeO}$ no apparent changes can be identified at $T_{\mathrm{N}}$, but at $5 \mathrm{~K}$ the reflectivity is roughly constant for $\omega<150 \mathrm{~cm}^{-1}$. Furthermore, the line shape of the $600 \mathrm{~cm}^{-1}$ anomaly has developed a two-peak structure. Contrarily, the cobalt compound offers a series of new excitations in the ordered phase. Beside a splitting of the TO modes, sharp antiresonance like anomalies become visible between 140 and $300 \mathrm{~cm}^{-1}$, which have convincingly be described as transitions between the spin-orbit and exchange split ground state of the $\mathrm{Co}^{2+}$-ion [46]. The narrow wiggles in the spectrum below $200 \mathrm{~cm}^{-1}$ are due to interference fringes and represent no bulk properties. The nickel compound finally, does not exhibit any significant new features. However, since $T_{\mathrm{N}}=523 \mathrm{~K}$ no structural changes are present between room temperature and $5 \mathrm{~K}$. The anomaly at $390 \mathrm{~cm}^{-1}$ becomes more pronounced and hardens similarly to the main and the two-phonon modes. Figure 3 documents 
an interesting aspect of the late TMOs. As will be shown in the following the width of the reststrahlen band is directly related to the effective charge involved in the vibrations, which should be close to $2 e$. However, the width changes considerably being largest in $\mathrm{MnO}$ and narrow in NiO. From this observation one can directly conclude that the ionic/covalent character of the bonds decreases/increases from $\mathrm{MnO} \rightarrow \mathrm{CoO} \rightarrow \mathrm{FeO} \rightarrow \mathrm{NiO}$.

Since the main interest of the present study is to investigate the interplay of the lattice dynamics with the spin degrees of freedom, the effects attributed to be purely of electronic origin will not be touched here. These have already been described in great detail for $\mathrm{CoO}$ in [46] or will be reported in a subsequent publication in case of $\mathrm{FeO}$. The detailed temperature dependence of the phonon properties for all investigated monoxides are reported in the following subsections. Since for $\mathrm{MnO}$ and $\mathrm{CoO}$ this has been already done thoroughly $[38,46]$, these results are presented briefly and the focus is put to the other compounds. The lattice excitations are studied by inspection of the complex dielectric function $\varepsilon(\omega)$ obtained via the Kramers-Kronig relation. Its imaginary part, the dielectric loss $\varepsilon_{2}$, peaks at the TO eigenfrequency $\omega_{\mathrm{TO}}[57]$, while the energy loss function $-\operatorname{Im}\left(\varepsilon^{-1}\right)$ is solely sensitive to the longitudinal response, where the maximum is located at the LO eigenfrequency $\omega_{\mathrm{LO}}$ [58]. Having determined both quantities the static dielectric constant $\varepsilon_{0}$ can be derived from the generalized Lyddane-Sach-Teller relation [59]

$$
\frac{\varepsilon_{0}}{\varepsilon_{\infty}}=\frac{\prod_{j} \omega_{\mathrm{LO}_{j}^{2}}^{2}}{\prod_{j} \omega_{\mathrm{TO}_{j}^{2}}},
$$

where $\varepsilon_{\infty}$ accounts for electronic polarizations above the phonon domain and $j$ runs over the total number of modes. Another interesting quantity is the effective ionic plasma frequency $\Omega$, which can be associated with the effective charges participating in the vibrations [60]:

$$
\Omega^{2}=\varepsilon_{\infty} \sum_{j}\left(\omega_{\mathrm{LO}_{j}^{2}}^{2}-\omega_{\mathrm{TO}_{j}^{2}}^{2}\right)=\frac{N}{V} \frac{\varepsilon_{\infty}}{\varepsilon_{\mathrm{vac}}} \frac{\left(Z^{*} e\right)^{2}}{\mu} .
$$

Here $N / V$ denotes the number of ion pairs per unit cell and $\varepsilon_{\text {vac }}$ the permittivity of free space. $Z^{*} e$ is the effective charge, which should be close to $2 e$ for the purely ionic TMOs and $\mu$ represents the reduced mass of the involved ions.

\section{1 $\mathrm{MnO}$}

In Fig. 4(a) and (b) the eigenfrequencies of the TO and LO modes are presented as a function of temperature. The corresponding insets display typical dielectric loss and energy-loss functions, which were consulted to determine the resonance frequencies. In the paramagnetic phase $\omega_{\mathrm{TO}}$ and $\omega_{\mathrm{LO}}$ are approximately constant at around $270 \mathrm{~cm}^{-1}$ and $557 \mathrm{~cm}^{-1}$, respectively. But more importantly, a clear splitting of the mode occurs, which can be associated with the magnetic phase transition. At $5 \mathrm{~K}$ three distinct TO modes are visible located at 263.2, 278.5 and $288.8 \mathrm{~cm}^{-1}$, thus the overall splitting amounts to more than $25 \mathrm{~cm}^{-1}$. Similar observations hold also for $\omega_{\mathrm{LO}}$. These findings must be compared with recent neutron scattering results, which reported a single mode close to $268 \mathrm{~cm}^{-1}$ at $300 \mathrm{~K}$, but two phonon branches at liquid helium temperature located at 268.6 and $293.6 \mathrm{~cm}^{-1}$ [9]. These data are also provided in Fig. 4.

The derived quantities $\varepsilon_{0}$ and $\Omega$ as determined from Eqs. (2) and (3) are plotted in Fig. 4(c). $\varepsilon_{\infty}$ was found to scatter around 4.87 with no systematic temperature dependence and was fixed to this average value for the further analysis. The plasma frequency is almost constant above and below $T_{\mathrm{N}}$ and amounts to about $1075 \mathrm{~cm}^{-1}$. The increase for $T<100 \mathrm{~K}$ in the magnetically ordered state is of the same order as the decrease of the unit cell volume due to exchange striction [23]. Hence, no remarkable charge transfer processes are accompanied with the phase transition. Calculating the ionic plasma frequency via Eq. (3) by assuming an ideal ionic valence of $Z= \pm 2$ for both ions, results in $\Omega=1873 \mathrm{~cm}^{-1}$. This is significantly higher than the experimentally observed value at room temperature and rather implies $Z^{*} / Z \approx 0.58$, which 


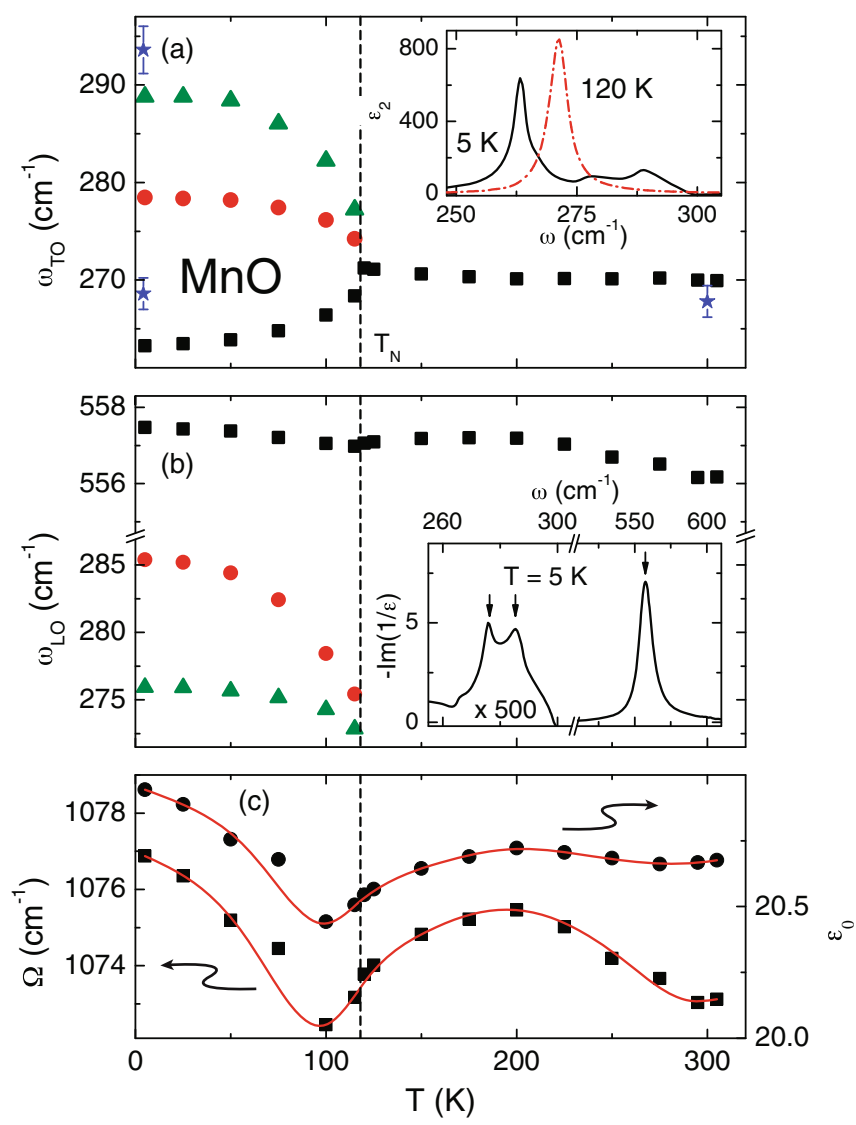

Fig. 4. Eigenfrequencies of the TO (a) and LO (b) phonon modes in $\mathrm{MnO}$ as determined from the corresponding insets. Arrows indicate peak position at $5 \mathrm{~K}$. Stars denote neutron scattering results [9]. (c) Derived quantities: plasma frequency (left scale) and static dielectric constant (right scale). Lines are drawn to guide the eye.

still signals predominantly ionic bonding, but with distinct covalent contributions. However, from $\gamma$-ray spectroscopy probing the electron density distribution purely ionic bondings have been identified [61]. A very similar evolution in temperature is found for $\varepsilon_{0}$ shown in Fig. 4(c). The average value is about 20.7 and the total change is of the order of $4 \%$.

\section{$3.2 \mathrm{FeO}$}

The reflectivity data of $\mathrm{FeO}$ have to be analyzed differently than the spectra of the other TMOs. Due to the comparably high conductivity of the order of $10 \Omega^{-1} \mathrm{~cm}^{-1}$ at room temperature [39] a Drude-like extrapolation have to be applied at low frequencies prior to the KramersKronig transformation. Furthermore, the effects of plasmon-phonon coupling hamper the direct evaluation of $\omega_{\mathrm{TO}}$ and $\omega_{\mathrm{LO}}$. Thus, it is more convenient to fit $\varepsilon(\omega)$ by the model dielectric function

$$
\varepsilon(\omega)=\varepsilon_{\infty}-\frac{\varepsilon_{\infty} \Omega_{P}^{2}}{\omega^{2}+\mathrm{i} \gamma_{P} \omega}+\sum_{j} \frac{\Omega_{j}^{2}}{\omega_{\mathrm{TO}}^{2}-\omega^{2}-\mathrm{i} \gamma_{j} \omega}
$$

as proposed in literature $[40,42]$. The second term on the right hand side represents a Drude like contribution and accounts for free charge carriers with plasma frequency $\Omega_{P}$ and damping $\gamma_{P}$, while each addend of the sum describes one normal mode according to a Lorentz oscillator. $\Omega_{j}$ is an effective ionic plasma frequency for the corresponding mode and $\gamma_{j}$ is the damping. Thus, there are three adjustable parameters per lattice vibration plus two for the free charge carrier response and one to account for electronic polarizations. 


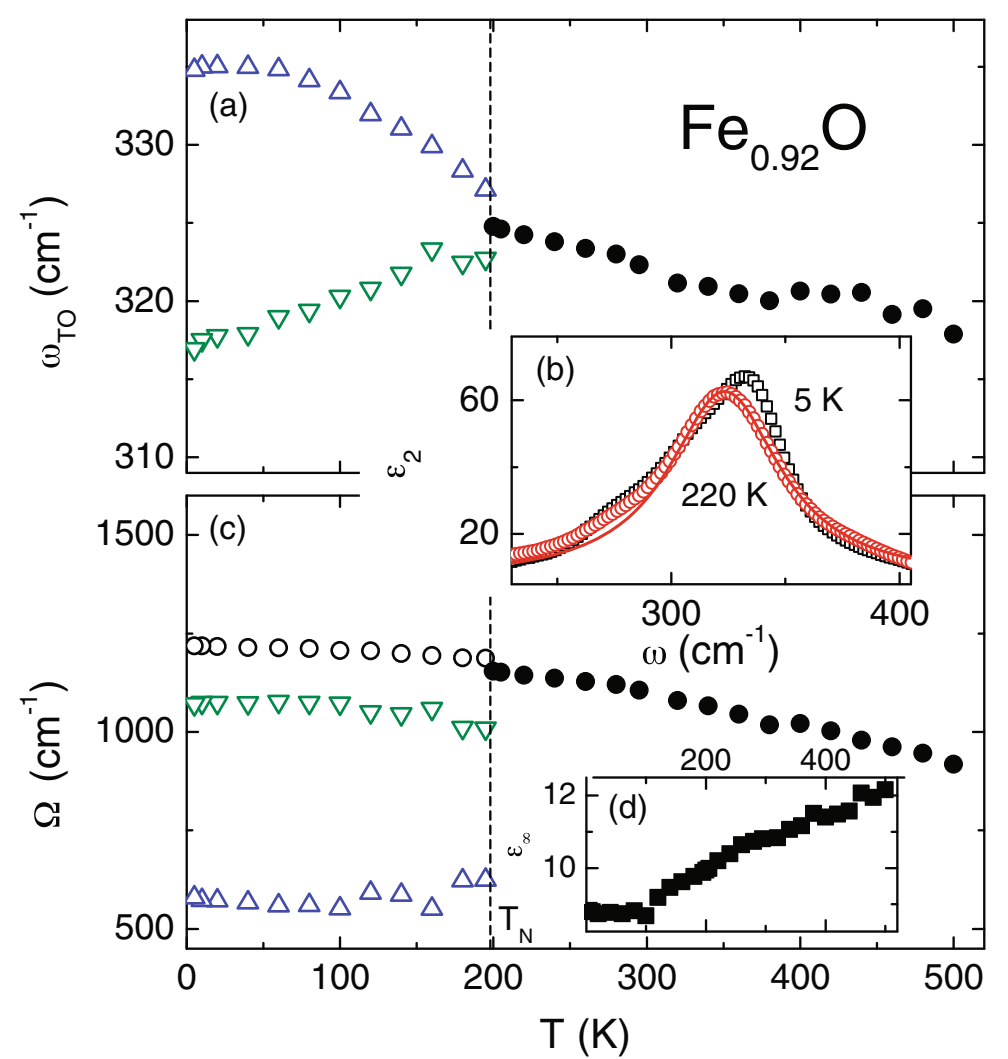

Fig. 5. (a) Eigenfrequency of the TO phonon mode in FeO. (b) Dielectric loss peak around the TO phonon mode at $220 \mathrm{~K}$ and $5 \mathrm{~K}$. Solid line is a Lorentzian fit to the $220 \mathrm{~K}$ data. (c) Effective ionic plasma frequency $\Omega$ for the phonon mode. Open circles below $T_{\mathrm{N}}$ dentotes the total plasma frequency of both modes. (d) $\varepsilon_{\infty}$ as a function of temperature.

Above $T_{\mathrm{N}}$ just one oscillator was regarded in Eq. (4) as expected for the cubic rock salt structure. However, as it is depicted in Fig. 5(b), the dielectric loss peak representing the TO phonon develops an increasing asymmetry in the ordered phase. At $220 \mathrm{~K}$ it can be fitted reasonably to a Lorentzian line shape, but at $5 \mathrm{~K}$ it seems that a second mode appears at higher frequencies. This observation is believed to be due to a splitting of the degenerate phonon mode in the distorted structure. However, due to the high damping of the order of $100 \mathrm{~cm}^{-1}$, both maxima cannot be identified unambiguously. Hence, to account for this behavior two oscillators were considered in Eq. (4) for $T<T_{\mathrm{N}}$.

The eigenfrequencies and ionic plasma frequencies obtained from fits using Eq. (4) are displayed in Fig. 5 as a function of temperature. In the paramagnetic state $\omega_{\text {TO }}$ hardens on cooling as expected for an ordinary anharmonic solid. Below $T_{\mathrm{N}}$ two modes smoothly develop from the triply degenerate one and are separated by almost $18 \mathrm{~cm}^{-1}$ at $5 \mathrm{~K}$. Nevertheless, it has to be stated here explicitly that the determination of the eigenfrequencies below $T_{\mathrm{N}}$ solely relies on the fitting procedure and that beside the asymmetry no indication for a split-off mode is apparent. However, it is reasonable to assume that the lower symmetric rhombohedral structure in the ordered state also alters the phonon properties. A direct evaluation of the peak maxima reveals a very similar dependence to the upper mode and also features a kink at $T_{\mathrm{N}}$. The effective ionic plasma frequencies of the modes (cf. Fig. 5(c)) are almost constant in the ordered state. The ratio amounts to approximately $2: 1$, which is consistent with a splitting into a doubly degenerate and a single mode. The total plasma frequency smoothly develops from the ordered to the paramagnetic state, where it decreases continuously on rising temperatures due to screening effects. The theoretically calculated value of $\Omega$, according to Eq. (3), is $2076 \mathrm{~cm}^{-1}$ 


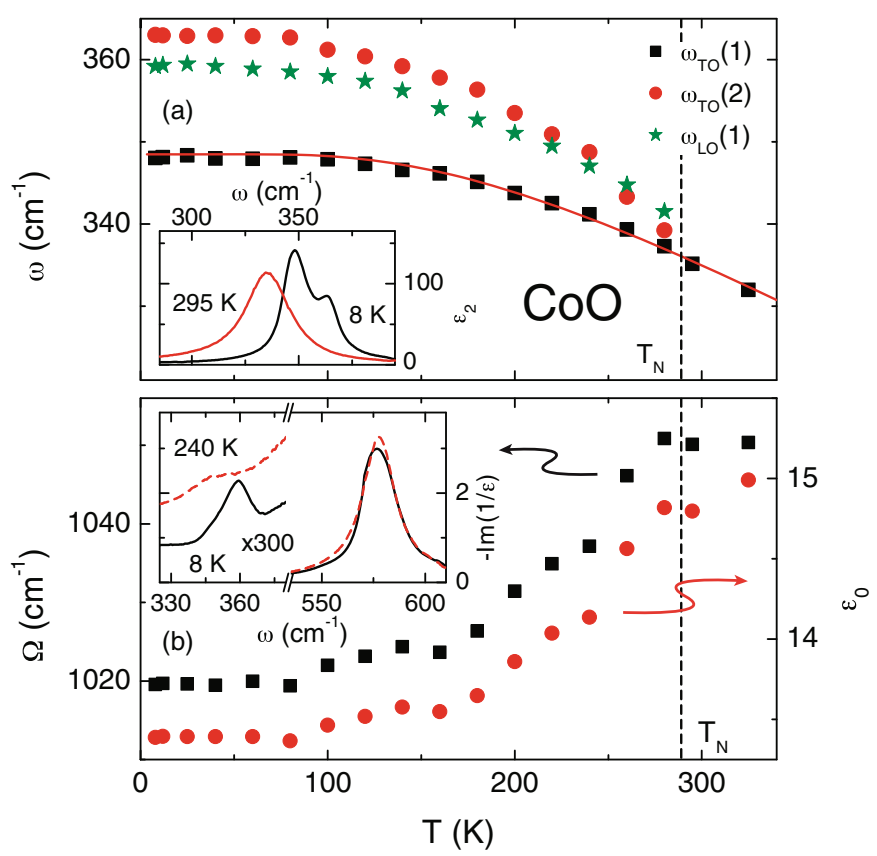

Fig. 6. (a) Temperature evolution of the eigenfrequencies of TO and the split-off LO modes. Solid line is a fit according to a simple anharmonic model (see text). (b) Effective ionic plasma frequency (squares, left scale) and static dielectric constant (circles, right scale). Also provided are the dielectric loss (upper inset) and the energy loss function (lower inset) around the resonance frequencies $\omega_{\text {TO }}$ and $\omega_{\mathrm{LO}}$, respectively.

for an idealized ionic bond. The significant lower value observed in Fig. 5(c) indicates already covalent contributions to the bondings at lowest temperatures, where screening effects from the plasmons should be negligible. The tiny step visible in Fig. 5 (c) at $T_{\mathrm{N}}$ is most probably due to the forced fitting. It should also be mentioned here that $\varepsilon_{\infty}$ was found to be strongly temperature dependent above $50 \mathrm{~K}$, where it increases linearly from 9 to about 12 at $500 \mathrm{~K}$ (cf. Fig. 5(d)). This might indicate the presence of a strongly temperature dependent crystal field excitation or a strong increase of conductivity contributions. However, this effect does not seem to be correlated with the magnetic transition.

\section{$3.3 \mathrm{CoO}$}

Figure 6 contains the eigenfrequencies of the TO modes in $\mathrm{CoO}$ determined by inspecting the peak positions of $\varepsilon_{2}$ for $8 \leq T \leq 325 \mathrm{~K}$. The single cubic vibration smoothly becomes the main phononic branch in the distorted structure below $T_{\mathrm{N}}$. At higher frequencies a second resonance splits off and gains strength. At $8 \mathrm{~K}$ the separation is maximum and amounts to almost $15 \mathrm{~cm}^{-1}$. Also presented are the resonance frequencies of the weaker LO mode, revealing a similar hardening like the TO excitations. The dominant LO response, however, stays almost constant at $577 \mathrm{~cm}^{-1}$ for all measured temperatures as documented in the lower inset of Fig. 6. The solid line describing the main TO mode is a fit according to the simple anharmonic model [62]

$$
\omega_{\mathrm{TO}}(T)=\omega_{0}\left(1-\frac{c}{\exp \left(\theta_{\mathrm{D}} / T\right)-1}\right)
$$

where $\omega_{0}$ indicates the eigenfrequency at $0 \mathrm{~K}$ and $c$ is a mode dependent scaling factor. The Debye temperature is found to yield $\theta_{\mathrm{D}}=582 \mathrm{~K}$.

From $\omega_{\mathrm{TO}}$ and $\omega_{\mathrm{LO}}$ the quantities $\Omega$ and $\varepsilon_{0}$ are derived and plotted in Fig. 6(b). Clearly visible is the striking parallel evolution with temperature. $\Omega$ is constant at $1050 \mathrm{~cm}^{-1}$ in the cubic phase and rapidly decreases below $T_{\mathrm{N}}$. It saturates for $T<100 \mathrm{~K}$ at about $1020 \mathrm{~cm}^{-1}$. 


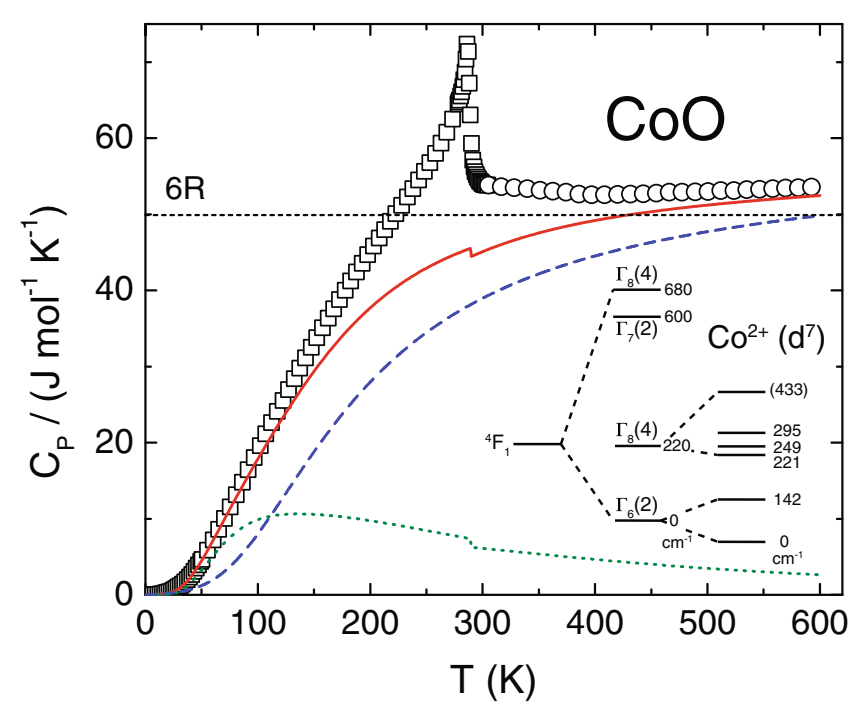

Fig. 7. Specific heat of $\mathrm{CoO}$ as a function of temperature. Open squares are obtained from measurements of the investigated sample, while open circles $(T>305 \mathrm{~K})$ are taken from [63]. The dashed line denotes the lattice contribution to the specific heat and the dotted one represents a Schottky anomaly induced by the ground state splitting indicated in the level scheme due to spin-orbit coupling and super exchange [46]. The solid line is a sum of both contributions and a small linear term (see text for details).

This $3 \%$ effect cannot be explained by the a decrease of the unit cell volume and consequently has to be ascribed to charge-transfer processes.

The molar specific heat for the investigated $\mathrm{CoO}$ sample is documented in Fig. 7. Clearly visible is a $\lambda$-like anomaly close to $289 \mathrm{~K}$. The data above $T=305 \mathrm{~K}$ are taken from [63] and smoothly extend our measurement. In the following these data shall be analyzed in more detail to estimate the underlying mechanisms contributing at this energy scale. As it was pointed out in [46] the crystal field split ground state ${ }^{4} F_{1}$ of the $\mathrm{Co}^{2+}$-ion further lifts its degeneracy due to spin-orbit coupling and below $T_{\mathrm{N}}$ additionally by super exchange. This is schematically shown in the level scheme of Fig. 7 , where the ${ }^{4} F_{1}$ term splits in the second column according to the former and in the third due to the latter effect. The numbers next to the levels are experimental values provided in [46] except for the one in parentheses, which was calculated. The thermal population of the electronic levels give rise to a Schottky-like anomaly in $C_{P}$ and is shown as dotted line in Fig. 7. The discontinuity at $T_{\mathrm{N}}$ arises from the vanishing influence of the magnetic exchange in the paramagnetic state. It should be mentioned, that the Schottky anomaly is fully determined by the values given in [46], though a slight temperature dependence has not been incorporated here. The lattice contribution can then be estimated by one isotropic Debye and one isotropic Einstein contribution with an Einstein temperature $\theta_{\mathrm{E}}=600 \mathrm{~K}$, which corresponds nicely to the location of the reststrahlen band, and a Debye temperature $\theta_{\mathrm{D}}=650 \mathrm{~K}$ being in excellent agreement with the findings in the isostructural $\mathrm{NiO}$, where $\theta_{\mathrm{D}}=653 \mathrm{~K}$ [64]. The solid line in Fig. 7 is a sum of both contributions and a small linear term, which accounts for the thermal expansion and is found to be of the order of $5 \mathrm{~mJ} \mathrm{~mol}^{-1} \mathrm{~K}^{-1}$ [65]. The obtained results nicely reproduces the experimental data at low and elevated temperatures. The intermediate region is dominated by the magnetic transition, and the deviations can be ascribed to magnetic contributions. Calculating the entropy due to the residual heat gives $10.5 \mathrm{~J} \mathrm{~mol}^{-1} \mathrm{~K}^{-1}$ at $600 \mathrm{~K}$, which is in good agreement to $R \ln 4=11.5 \mathrm{~J} \mathrm{~mol}^{-1} \mathrm{~K}^{-1}$ expected for a $S=3 / 2$ system, especially when having in mind the rough approximations used in this evaluation.

\section{$3.4 \mathrm{NiO}$}

The eigenfrequencies of the TO and weaker LO mode as determined by evaluation of the peak maxima of $\varepsilon_{2}$ and $-\operatorname{Im}\left(\varepsilon^{-1}\right)$ are plotted in Fig. 8(a). The overall evolution is very similar to the 


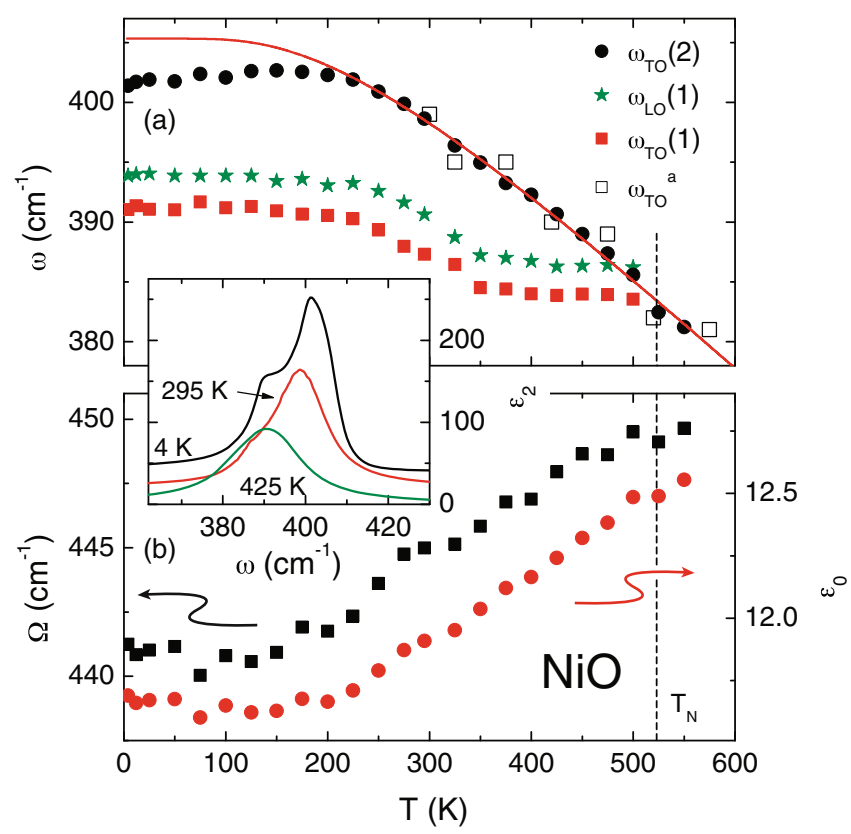

aS. Mochizuki and M. Satoh, Ref. [50]

Fig. 8. (a) Mode frequencies of the TO and weaker LO resonances for $4 \leq T \leq 550 \mathrm{~K}$. Open squares denote results from [50]. The solid line is a fit according to Eq. (5). (b) $\Omega$ (squares, left scale) and $\varepsilon_{0}$ (circles, right scale) determined from the phonon properties. Inset: Dielectric loss around the TO modes for various temperatures.

one observed in $\mathrm{CoO}$. The cubic mode above $T_{\mathrm{N}}$ continuously develops into the main resonance of the ordered phase and can well be described by Eq. (5) assuming a Debye temperature of $\theta_{\mathrm{D}}=636 \mathrm{~K}$ down to $200 \mathrm{~K}$. Also provided are the results of emission spectroscopy measurements [50] being in excellent agreement with the obtained parameters at elevated temperatures. However, the split-off mode appears at lower frequencies in this case with an anomalous temperature dependence. It stays almost constant at $384 \mathrm{~cm}^{-1}$ before anharmonic effects dominate below room temperature. For $T<200 \mathrm{~K}$ the eigenfrequency finally saturates close to $391 \mathrm{~cm}^{-1}$. This behavior can qualitatively be explained by competing interactions due to spin-spin correlations and an ordinary lattice hardening. The major LO mode is found to be almost temperature independent with $\omega_{\mathrm{LO}}=595 \mathrm{~cm}^{-1}$. Only at high temperatures it starts to soften by an amount of roughly $1 \%$ at $525 \mathrm{~K}$. The weaker LO resonance shows a very similar dependence to the corresponding TO mode. For all temperatures the eigenfrequencies are shifted constantly by about $3 \mathrm{~cm}^{-1}$. However, on approaching $T_{\mathrm{N}}$ it gets increasingly difficult to determine the exact peak positions of the weaker modes as can be pictured from the inset of Fig. 8.

In the lower frame of Fig. 8 the calculated plasma frequency and static dielectric constant are plotted. During the evaluation, an average value of $\varepsilon_{\infty}=5.25$ was taken, since no systematic temperature dependence could be identified. Both quantities exhibit a very similar temperature dependence to the corresponding ones in $\mathrm{CoO}$. $\Omega$ is almost constant at $441 \mathrm{~cm}^{-1}$ and increases between 150 and $550 \mathrm{~cm}^{-1}$ almost linearly up to $450 \mathrm{~cm}^{-1}$. Unfortunately, the measurements do not allow to identify a clear trend for even higher $T$, although the data of both quantities seem to saturate above $T_{\mathrm{N}}$. The plasma frequency of $\approx 2100 \mathrm{~cm}^{-1}$ expected for $Z^{*} e=2 e$ from Eq. (3) is not reached. This discrepancy indicate strong covalent contributions in the bondings of $\mathrm{NiO}$. The static dielectric constant decreases in the magnetically ordered state from 12.5 by about $5 \%$ at liquid helium temperatures with an evolution comparable to that of the ionic plasma frequency $\Omega$.

The reflectivity of $\mathrm{NiO}$ over a broad frequency range $\left(200 \leq \omega \leq 40000 \mathrm{~cm}^{-1}\right)$ is documented in Fig. 9 for selected temperatures. Beyond the phonon domain, above $1000 \mathrm{~cm}^{-1}$ 


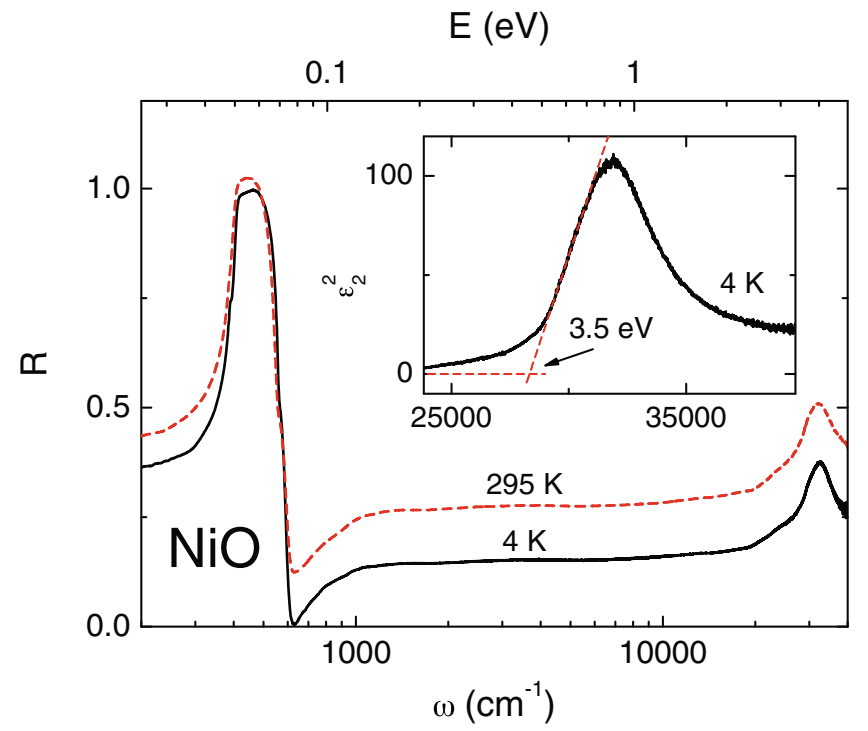

Fig. 9. Reflectivity of $\mathrm{NiO}$ for $200 \leq \omega \leq 40000 \mathrm{~cm}^{-1}$ at 4 and $295 \mathrm{~K}$ (shifted for clarity). Inset: $\varepsilon_{2}^{2}$ vs. $\omega$ around the fundamental transition at $4 \mathrm{~K}$. The linear extrapolation leeds to $E_{\mathrm{Gap}} \approx 3.5 \mathrm{eV}$.

an almost constant and featureless reflectivity plateau extends to $20000 \mathrm{~cm}^{-1}$. The reflectivity exhibits a peak close to $32500 \mathrm{~cm}^{-1}$, which results from charge transfer transitions and does not show a pronounced temperature dependence. Plotting the squared dielectric loss $\varepsilon_{2}^{2}$ as a function of frequency reveals a striking linear dependence of the low frequency edge as is depicted for the $4 \mathrm{~K}$ data in the inset of Fig. 9. From a linear extrapolation of the slop to $\varepsilon_{2}^{2}=0$, the gap value is estimated being $E_{\text {Gap }}=3.5 \mathrm{eV}$ with no systematic temperature evolution. This kind of analysis, however, suggests a direct absorption edge. The value is in good agreement with previous optical reflectivity and ellipsometry measurements reporting 3.87 [66] and $3.7 \mathrm{eV}[67]$, respectively. These results correspond nicely to the inverse photoemission spectra of Zimmermann et al. [68], where the lowest conduction band peaks at $\approx 3.65 \mathrm{eV}$. The gap energy determined in [69] by means of combined x-ray photoemission and bremsstrahlungisochromat spectroscopy, however, seems to be significantly enhanced with $E_{\text {Gap }}=4.3 \mathrm{eV}$. It is not easy to explain the discrepancies in the reported gap energies, but they could reflect the different probing techniques or indicate variations in the sample stoichiometries or sample qualities.

\section{Discussion and concluding remarks}

As outlined above, the AFM ordering is accompanied by structural distortions in all TMOs. Due to the accompanying symmetry reduction, a splitting of the degenerate $T_{1 u}$ mode is expected. Knowing the type of lattice distortions in the ordered state, the conceptual simple rock-salt structure alleviates the prediction of the number of expected phonon modes. In the case of $\mathrm{MnO}, \mathrm{FeO}$ and $\mathrm{NiO}$ featuring rhombohedral symmetry in the low-temperature modification, the only compatible subgroup of the $F m \overline{3} m$ space group is $R \overline{3} m$ (\#166) [70]. Assuming further that the high-symmetry Wyckoff positions $1 a$ and $1 b$ of the rhombohedral unit cell are occupied, which correspond to the corner and body center positions, the mechanical representation becomes [71]

$$
\Gamma_{\text {mech }}=\underbrace{A_{2 u}+E_{u}}_{\text {acoustic }}+\underbrace{A_{2 u}+E_{u}}_{\text {IR active }} .
$$

Thus, a splitting into two subsequent excitations is estimated where one is a singlet and the other a doublet. The assignment of the crystallographic symmetry is in accordance with the 
reports in $[72]$ for $\mathrm{NiO}$. In the case of $\mathrm{CoO}$ with a monoclinic symmetry, the reported distorted space group is $C 2 / m$ (\#12) with two Co-ions in $2 a$ and two O-ions in $2 d$ positions [32]. For this arrangement the mechanical representation reads as [71]

$$
\Gamma_{\text {mech }}=\underbrace{A_{u}+2 B_{u}}_{\text {acoustic }}+\underbrace{A_{u}+2 B_{u}}_{\text {IR active }}
$$

and, hence, three individual resonances can be expected in the optical spectra of $\mathrm{CoO}$ in the magnetically ordered phase.

On the basis of these factor group analyses, the experimentally determined phonon excitations can be discussed. First, in all four monoxides a clear anisotropy in the phonon properties has been identified, which becomes apparent below $T_{\mathrm{N}}$ and increases in strength upon further cooling. The overall splitting $\Delta \omega$ of the modes becomes less pronounced going through the series of the investigated TMOs. At the lowest measured temperature, it amounts to $25.6 \mathrm{~cm}^{-1}$ in $\mathrm{MnO}, 17.9 \mathrm{~cm}^{-1}$ in $\mathrm{FeO}, 15.0 \mathrm{~cm}^{-1}$ in $\mathrm{CoO}$ and $10.3 \mathrm{~cm}^{-1}$ in $\mathrm{NiO}$, respectively. Comparing these with neutron scattering results [9] for $\mathrm{MnO}$ and $\mathrm{NiO}$ reporting $\Delta \omega=25 \mathrm{~cm}^{-1}$ and $40 \mathrm{~cm}^{-1}$, respectively, the agreement seems to be reasonable in the first but overestimated in the latter compound. From LSDA calculations Luo et al. [8] estimate a splitting of $\Delta \omega=30.6 \mathrm{~cm}^{-1}$ and $14.5 \mathrm{~cm}^{-1}$ in these compounds, which is close to our results. It should be noted, that in the referenced studies only two resonances could be identified in $\mathrm{MnO}$, which is in contrast to the present investigation, where three modes could clearly be detected. However, the pattern of the splittings for the TMOs is not in accordance with the proposed low-temperature symmetry in each case. While $\mathrm{FeO}$ and $\mathrm{NiO}$ offer the expected splitting in two individual vibrations with a distribution of the optical weight close to $2: 1, \mathrm{MnO}$ and $\mathrm{CoO}$ disobey the predictions. The observation of three modes in $\mathrm{MnO}$ is indicative of a lower symmetry than rhombohedral as it was already pointed out in [22]. In $\mathrm{CoO}$ some disagreement with theory was found, instead of three only two could be identified unambiguously. This might be due to the fact that the induced splitting is too weak, so that it cannot be identified in the present study, or that two modes are accidentally degenerate.

Inspecting the different splitting patterns of the TMOs among each other, a further remarkable observation can be made. When looking at the temperature dependence of the main resonance in $\mathrm{CoO}$ and $\mathrm{NiO}$, they seem to be very little influenced by the magnetic ordering but can be well described by the simple anharmonic model of Eq. (5) above and below $T_{\mathrm{N}}$ with reasonable $\theta_{\mathrm{D}}$. Contrary, in the two other monoxides $\mathrm{MnO}$ and $\mathrm{FeO}$ all resonances in the ordered state seem to move apart from the formerly cubic TO excitation, so that a clear discontinuity occurs at $T_{\mathrm{N}}$ for all eigenfrequenies. Following the arguments of Luo et al. [8] the singlet mode is polarized along the magnetic ordering direction, while the other two are degenerate and perpendicular to the former one. The energy sequence is found to depend on the sign of the exchange integral between the frustrated nearest neighbors $J_{1}$. This is consistent with a split-off mode located at higher frequencies in $\mathrm{MnO}\left(J_{1}=0.86 \mathrm{meV}\right.$ [73] $)$ and a weaker excitation occurring below the main phonon mode in $\mathrm{NiO}\left(J_{1}=-0.69 \mathrm{meV}[74]\right)$. However, it should be mentioned that the frustrated $J_{1}$ coupling is less well determined in both sign and size than the antiferromagnetic $J_{2}$ exchang from the experimental point of view. A direct comparison of $\Delta \omega$ and $J_{1}$ is thus challenged due to this puzzling situation in the late TMOs. As a consequence the present investigation might contribute to clarify these discrepancies.

The quantities $\Omega$ and $\varepsilon_{0}$ derived from the lattice excitations are found to be rather constant in $\mathrm{MnO}$ over the whole temperature range. The effective plasma frequency reduces continuously at elevated temperatures owing screening processes due to the rising conductivity. $\mathrm{CoO}$ and $\mathrm{MnO}$ exhibit a rather similar dependence in $\Omega$ and $\varepsilon_{0}$. Above $T_{\mathrm{N}}$ both quantities are almost constant and rapidly decrease on entering the ordered state where they finally saturate at low temperatures. The decrease of the effective plasma frequencies can be attributed to significant charge transfer processes.

For the cobalt compound the specific heat data were analyzed in detail. Taking into account a Schottky anomaly induced by orbital degrees of freedom and modeling the phonon density of state by one Debye- and one Einstein-like term, the experimental data could be nicely reproduced revealing the magnetic contributions. 
Table 3. Optical properties of the investigated TMOs at room temperature. For FeO $\omega_{\mathrm{LO}}$ was obtained from the Lyddane-Sachs-Teller relation. The data of $\mathrm{NiO}$ refer to the main phonon excitation.

\begin{tabular}{cccccc}
\hline & $\omega_{\text {TO }}\left(\mathrm{cm}^{-1}\right)$ & $\omega_{\mathrm{LO}}\left(\mathrm{cm}^{-1}\right)$ & $\Omega\left(\mathrm{cm}^{-1}\right)$ & $\varepsilon_{0}$ & $\varepsilon_{\infty}$ \\
\hline $\mathrm{MnO}$ & 267.0 & 556.2 & 1073 & 20.7 & 4.87 \\
$\mathrm{FeO}$ & 322.3 & 466.0 & 1106 & 22.6 & 10.80 \\
$\mathrm{CoO}$ & 335.2 & 577.7 & 1050 & 14.8 & 4.98 \\
$\mathrm{NiO}$ & 398.6 & 595.3 & 445 & 11.9 & 5.25 \\
\hline
\end{tabular}

The phononic and optical properties at room temperature as determined in the present study are summarized in Tab. 3. The TO eigenfrequencies strongly increase in the series of the investigated TMOs. This fact must result from increasing force constants probably driven by an increasing covalency. The nature of the bonds also manifests in the values of the effective ionic plasma frequencies. As indicated in Eq. (3) $\Omega$ is directly related to the effective charges. Inspecting Tab. 3 the large effective ionic plasma frequency of $\mathrm{FeO}$ cannot be understood and obviously results from the unusual high value of $\varepsilon_{\infty}$. This large $\varepsilon_{\infty}$ stems from low lying crystal field excitations and from the influence of free charge carriers in non-stoichiometric $\mathrm{FeO}$.

Concerning the main observation of a splitting of the zone center optical modes in all investigated compounds, it is unclear whether this effect arises from ordinary lattice distortions or is due to the non-cubic spin density distribution. A detailed microscopic theory as in the case of frustrated pyrochlore lattices $[17,18]$ is demanded. For $\mathrm{MnO}$ it was shown [7] that lattice distortions can account only for deviations in the order of $1 \%$. However, detailed lattice dynamical calculations for the other TMOs are not available. Therefore, the splittings ranging between 3 and 10\% might be interpreted in the framework of the spin-driven Jahn-Teller transition.

We thank D. Vieweg and A. Pimenova for technical support. This work was supported by the Deutsche Forschungsgemeinschaft (DFG) through the Collaborative Research Center SFB 484 (University of Augsburg).

\section{References}

1. C.G. Shull, J.S. Smart, Phys. Rev. 76, 1256 (1949)

2. L. Nèel, Ann. Phys. 17, 5 (1932)

3. J.H. de Boer, E.J.W. Verwey, Proc. Phys. Soc. London 49, 59 (1937)

4. N.F. Mott, R. Peierls, Proc. Phys. Soc. London 49, 72 (1937)

5. J. Zaanen, G.A. Sawatzky, J.W. Allen, Phys. Rev. Lett. 55, 418 (1985)

6. C. Rödl, F. Fuchs, J. Furthmüller, F. Bechstedt, Phys. Rev. B 79, 235114 (2009)

7. S. Massidda, M. Posternak, A. Baldereschi, R. Resta, Phys. Rev. Lett. 82, 430 (1999)

8. W.D. Luo, P.H. Zhang, M.L. Cohen, Solid State Commun. 142, 504 (2007)

9. E.M.L. Chung, D.M. Paul, G. Balakrishnan, M.R. Lees, A. Ivanov, M. Yethiraj, Phys. Rev. B 68, 140406(R) (2003)

10. T. Rudolf, Ch. Kant, F. Mayr, J. Hemberger, V. Tsurkan, A. Loidl, New J. Phys. 9, 76 (2007)

11. T. Rudolf, Ch. Kant, F. Mayr, J. Hemberger, V. Tsurkan, A. Loidl, Phys. Rev. B 76 (2007)

12. T. Rudolf, Ch. Kant, F. Mayr, M. Schmidt, V. Tsurkan, J. Deisenhofer, A. Loidl, Eur. Phys. J. B 68, $153(2009)$

13. Ch. Kant, J. Deisenhofer, V. Tsurkan, A. Loidl, J. Phys.: Conf. Ser. 200, 032032 (2009)

14. S. Bordács, D. Varjas, I. Kézsmárki, G. Mihály, L. Baldassarre, A. Abouelsayed, C.A. Kuntscher, K. Ohgushi, Y. Tokura, Phys. Rev. Lett. 103, 077205 (2009)

15. T. Rudolf, Ch. Kant, F. Mayr, J. Hemberger, V. Tsurkan, A. Loidl, Phys. Rev. B 75, 052410 (2007)

16. Y. Yamashita, K. Ueda, Phys. Rev. Lett. 85, 4960 (2000)

17. O. Tchernyshyov, R. Moessner, S.L. Sondhi, Phys. Rev. Lett. 88, 067203 (2002)

18. O. Tchernyshyov, R. Moessner, S.L. Sondhi, Phys. Rev. B 66, 064403 (2002) 
19. W.L. Roth, Phys. Rev. 110, 1333 (1958)

20. L.S. Darken, R.W. Gurry, J. Am. Chem. Soc. 68, 798 (1946)

21. C.A. McCammon, J. Magn. Magn. Mater. 104, 1937 (1992)

22. H. Shaked, J. Faber, R.L. Hitterman, Phys. Rev. B 38, 11901 (1988)

23. B. Morosin, Phys. Rev. B 1, 236 (1970)

24. R.W. Tyler, Phys. Rev. 44, 776 (1933)

25. A.L. Goodwin, M.G. Tucker, M.T. Dove, D.A. Keen, Phys. Rev. Lett. 96, 047209 (2006)

26. C.A. McCammon, L.G. Liu, Phys. Chem. Miner. 10, 106 (1984)

27. F.B. Koch, M.E. Fine, J. Appl. Phys. 38, 1470 (1967)

28. N.C. Tombs, H.P. Rooksby, Nature 165, 442 (1950)

29. C.G. Shull, W.A. Strauser, E.O. Wollan, Phys. Rev. 83, 333 (1951)

30. H. Bizette, Ann. Phys. 1, 295 (1946)

31. J.R. Singer, Phys. Rev. 104, 929 (1956)

32. S. Saito, K. Nakahigashi, Y. Shimomura, J. Phys. Soc. Jpn. 21, 850 (1966)

33. L.C. Bartel, B. Morosin, Phys. Rev. B 3, 1039 (1971)

34. H.P. Rooksby, Nature 152, 304 (1943)

35. H. Kondoh, T. Takeda, J. Phys. Soc. Jpn. 19, 2041 (1964)

36. J.N. Plendl, L.C. Mansur, S.S. Mitra, I.F. Chang, Solid State Commun. 7, 109 (1969)

37. S. Mochizuki, J. Phys.: Condens. Matter 1, 10351 (1989)

38. T. Rudolf, Ch. Kant, F. Mayr, A. Loidl, Phys. Rev. B 77, 024421 (2008)

39. H. Bowen, D. Adler, B. Auker, J. Solid State Chem. 12, 355 (1975)

40. B. Prévot, J. Biellmann, M.F. Meftah, M. Sieskind, Phys. Stat. Sol. A 40, 503 (1977)

41. T. Henning, H. Mutschke, Astron. Astrophys. 327, 743 (1997)

42. C.T. Seagle, W. Zhang, D.L. Heinz, Z. Liu, Phys. Rev. B 79, 014104 (2009)

43. G. Kugel, C. Carabatos, B. Hennion, B. Prevot, A. Revcolevschi, D. Tocchetti, Phys. Rev. B 16, $378(1977)$

44. P.J. Gielisse, J.N. Plendl, L.C. Mansur, R. Marshall, S.S. Mitra, R. Mykolajewycz, A. Smakula, J. Appl. Phys. 36, 2446 (1965)

45. I.G. Austin, E.S. Garbett, J. Phys. C: Solid State Phys. 3, 1605 (1970)

46. Ch. Kant, T. Rudolf, F. Schrettle, F. Mayr, J. Deisenhofer, P. Lunkenheimer, M.V. Eremin, A. Loidl, Phys. Rev. B 78, 245103 (2008)

47. J. Sakurai, W.J.L. Buyers, R.A. Cowley, G. Dolling, Phys. Rev. 167, 510 (1968)

48. Z. Yamani, W. Buyers, R. Cowley, D. Prabhakaran, Physica B 403, 1406 (2008)

49. R. Newman, R.M. Chrenko, Phys. Rev. 114, 1507 (1959)

50. S. Mochizuki, M. Satoh, Phys. Stat. Sol. B 106, 667 (1981)

51. J.L. Shapiro, B.F. Woodfield, R. Stevens, J. Boerio-Goates, M.L. Wilson, J. Chem. Thermodyn. 31, $725(1999)$

52. P.S. Silinsky, M.S. Seehra, Phys. Rev. B 24, 419 (1981)

53. M.S. Jagadeesh, M.S. Seehra, Phys. Rev. B 21, 2897 (1980)

54. N. Tristan, V. Zestrea, G. Behr, R. Klingeler, B. Buchner, H.A.K. von Nidda, A. Loidl, V. Tsurkan, Phys. Rev. B 77, 094412 (2008)

55. M. Born, M. Blackman, Z. Phys. 82, 37 (1933)

56. K.S. Upadhyaya, R.K. Singh, J. Phys. Chem. Solids 35, 1175 (1974)

57. K. Wakamura, T. Ogawa, T. Arai, J. Appl. Phys. 19, 249 (1980)

58. K. Wakamura, T. Arai, K. Kudo, J. Phys. Soc. Jpn. 40, 1118 (1976)

59. A.S. Barker, Phys. Rev. A 136, A1290 (1964)

60. J.F. Scott, Phys. Rev. B 4, 1360 (1971)

61. W. Jauch, M. Reehuis, Phys. Rev. B 67, 184420 (2003)

62. Ch. Kant, J. Deisenhofer, F. Mayr, F. Schrettle, A. Loidl, V. Gnezdilov, D. Wulferding, P. Lemmens, V. Tsurkan, Phys. Rev. B 80, 214417 (2009)

63. E.N. Abarra, K. Takano, F. Hellman, A.E. Berkowitz, Phys. Rev. Lett. 77, 3451 (1996)

64. R.A. Coy, C.W. Tompson, E. Gurmen, Solid State Commun. 18, 845 (1976)

65. R.J. Radwanski, Z. Ropka (2009) [arXiv:0901.0437]

66. T.D. Kang, H.S. Lee, H. Lee, J.Korean Phys. Rev. 50, 632 (2007)

67. R.J. Powell, W.E. Spicer, Phys. Rev. B 2, 2182 (1970)

68. R. Zimmermann, P. Steiner, R. Claessen, F. Reinert, S. Hüfner, P. Blaha, P. Dufek, J. Phys.: Condens. Matter 11, 1657 (1999)

69. G.A. Sawatzky, J.W. Allen, Phys. Rev. Lett. 53, 2339 (1984) 
70. M.I. Aroyo, J.M. Perez-Mato, C. Capillas, E. Kroumova, S. Ivantchev, G. Madariaga, A. Kirov, H. Wondratschek, Z. Krist. 221, 15 (2006)

71. E. Kroumova, M.I. Aroyo, J.M. Perez-Mato, A. Kirov, C. Capillas, S. Ivantchev, H. Wondratschek, Phase Transit. 76, 155 (2003)

72. M. Fiebig, D. Fröhlich, T. Lottermoser, V.V. Pavlov, R.V. Pisarev, H.J. Weber, Phys. Rev. Lett. 87, $137202(2001)$

73. M.E. Lines, E.D. Jones, Phys. Rev. A 139, A1313 (1965)

74. M.T. Hutchings, E.J. Samuelsen, Phys. Rev. B 6, 3447 (1972) 